\title{
Photonic Thermometry at PTB - Promising First Results for Contact Temperature Metrology Utilizing Optical Sensors
}

\author{
Stephan Krenek ${ }^{1}$, René Eisermann ${ }^{1}$, Georg Winzer ${ }^{2}$, Tobias Habisreuther ${ }^{3}$, Daniel Schmid ${ }^{1}$, \\ Hoang Viet Lai ${ }^{1}$, Steffen Rudtsch ${ }^{1}$ \\ 1 Physikalisch-Technische Bundesanstalt, Abbestr. 2 - 12, 10587 Berlin, Germany \\ 2 Leibniz-Institut für innovative Mikroelektronik, Im Technologiepark 25, 15236 Frankfurt/O., Germany \\ ${ }^{3}$ Leibniz-Institut für Photonische Technolgien (IPHT), Albert-Einstein-Str. 9, 07745 Jena, Germany \\ stephan.krenenk@ptb.de
}

\begin{abstract}
Summary:
Photonic sensors offer new possibilities for the metrological temperature determination in specific applications including high electric fields or harsh environments. Within two EU projects the PTB develop and validate different photonic thermometers from $0{ }^{\circ} \mathrm{C}$ to over $1500{ }^{\circ} \mathrm{C}$. The aim of this work is to develop and validate novel accurate photonic thermometers with uncertainties in the $\mathrm{mK}$ range and sensors for application within harsh environments at high temperatures. We show first results using silicon ring resonators which achieve high quality factors $(Q \approx 160000)$ and over $15 \mathrm{~dB}$ filter contrast.
\end{abstract}

Keywords: photonic thermometry, temperature sensor, fiber Bragg grating, ring resonator, photonic integrated circuit

\section{Introduction}

In the industrially most relevant temperature range from about $-100{ }^{\circ} \mathrm{C}$ to $1000{ }^{\circ} \mathrm{C}$ temperature measurements are commonly based on the measurement of electrical resistance (e.g. Pt100) or voltage (thermocouple). Photonic sensors, in contrast, use the light-matter interaction to measure temperature or strain with the additional advantage of a metal free, chemical inert and mechanical robust sensor design. For example, a change of the refractive index due to temperature results in a resonance wavelength shift of an optical resonator. Fibre optical sensors using Bragg gratings (FBG) or distributed fibre optic methods are commercially available and are highly promising for industrial monitoring applications [1]. Approaches using photonic thermometers show great potential to reach measurement uncertainties comparable to conventional electrical sensors [2,3]. Nevertheless, the determination and reduction of temperature uncertainty is still a challenging task.

The Physikalisch Technische Bundesanstalt (PTB) is working on special photonic sensors for temperature measurement within two European research projects.

The first project "Enhancing process efficiency through improved temperature measurement 2" (EMPRESS 2) has the overall aim of improving the efficiency of key industrial manufacturing processes through improved temperature measurement and control. The project focuses on accurate and $\mathrm{SI}$ traceable temperature measurement with different stable, reliable, durable and robust sensors. One objective is the introduction of traceable fibre optic measurements at high temperatures above $500{ }^{\circ} \mathrm{C}$. PTB is working together with the Leibniz-Institut für Photonische Technolgien (IPHT) on FBGs in sapphire fibres which can be used at temperatures exceeding $1500{ }^{\circ} \mathrm{C}$.

The second project "Photonic and Optomechanical Sensors for Nanoscaled and Quantum Thermometry" (PhotOQuant) deals with fundamental research for high-precision or primary temperature sensors. Two micrometre-sized chip-based techniques are designed, manufactured, characterized and calibrated: optomechanical sensors, in which the temperaturedependent Brownian motion of nanostructures is optically detected, and photonic resonators, in which planar waveguides (e.g. ring resonators) allow very high-resolution measurements of the refractive index change. Together with the Leibniz-Institut für innovative Mikroelektronik (IHP), PTB investigates $\mathrm{Si} / \mathrm{SiO}_{2}$ structures which are manufactured by standardized masking processes. 


\section{Perspective and First Results}

Our efforts are concentrated in two directions, first, the validation and improvement of photonic resonators (PhotoQuant) for moderate environments and temperatures up to $100^{\circ} \mathrm{C}$ with measurement uncertainties below $10 \mathrm{mK}$. Secondly, the metrological characterization of sapphire based fibre Bragg gratings (SFBG) for applications in harsh environments with temperatures above $1500^{\circ} \mathrm{C}$ (EMPRESS 2). Both photonic thermometer principles are mainly based on the determination of frequency (or wavelength) changes of an optical resonance.

Photonic ring resonators offer a high temperature coefficient $(\approx 73 \mathrm{pm} / \mathrm{K})$ together with a very high quality factor of the resonant frequency. In this work a photonic integrated circuit with over 130 resonator structures on a $2 \mathrm{~mm} \times 2 \mathrm{~mm}$ chip were manufactured with IHP's BICMOS silicon photonic platform [4] on a standard multi project wafer. The resonators were characterised using a tuneable laser with a tuning range from $1520 \mathrm{~nm}$ to $1630 \mathrm{~nm}$, variable gain preamplified photodiodes and a highly stable thermostat stage. Reference gas cells ( $\mathrm{HCN}$ and $\mathrm{C}_{2} \mathrm{H}_{2}$ ) were used for an in-situ SI traceable wavelength calibration [5]. Figure 1 shows an example of the normalised transmissions spectrum of three ring resonators with rip waveguide design. All three resonator are coupled to a single bus waveguide, but with different spacing between bus and ring. Each ring resonators have a radius of $r \approx 35 \mu \mathrm{m}$, resulting in a free spectral range of $F S R \approx 3 \mathrm{~nm}$. Due to the long tuning range of the laser it is possible to track the temperature shift of up to 50 resonance peaks simultaneously. In the lower part of Fig. 1 the shift of one peak per resonator is depicted. The resonators achieve very high quality factors of $Q \approx 160000$ and strong filter contrasts of $\Delta T \approx-15 \mathrm{~dB}$. These outstanding values for temperature applications [6-8], enable a very precise determination of the resonance wavelength. According to our preliminary results our photonic integrated circuits are potential suitable for temperature uncertainty below $10 \mathrm{mK}$.

Another solution suitable for even higher temperature above $1500^{\circ} \mathrm{C}$ (probably up to $1900{ }^{\circ} \mathrm{C}$ ) are Sapphire-FBG's, which offer a temperature coefficient of $26 \mathrm{pm} / \mathrm{K}$ [9]. Since they are intrinsically multimodal the corresponding resonance spectra is more complex compared to conventional single mode FBG [9]. Due to that, the precise determination of wavelength shift is the limiting factor for temperature uncertainties ( $1 \mathrm{~K}$ region), which is still under investigation.
In summary, photonic thermometry sensors are suitable to compete with conventional electrical sensors, however some effort and research have to be done in the future.

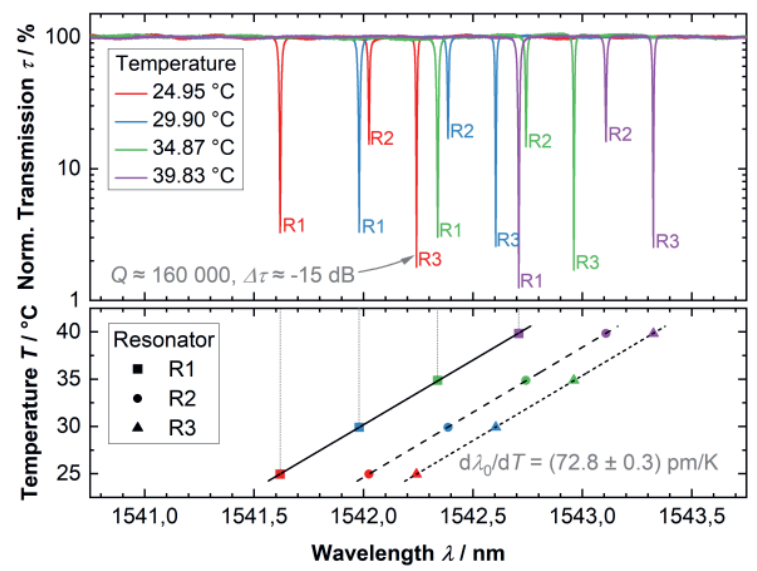

Fig. 1. Normalised Transmission of three ring resonators and temperature dependant shift of the resonance wavelength.

\section{Acknowledgment}

This work has received funding from the EMPIR programme co-financed by the Participating States and from the European Union's Horizon 2020 research and innovation programme.

\section{References}

[1] K. Hicke et al. Proc. SPIE 10323, 25th International Conference on Optical Fiber Sensors, 103230J (2017), doi: 10.1117/12.2272463.

[2] N. Klimov et al. Sensors Actuators A: Physical, 269, 308 (2018), doi: 10.1016/j.sna.2017.11.055

[3] Z. Ahmed, et al., Proc. SPIE 10923, Silicon Photonics XIV, 109230L (2019), doi:10.1117/12.2505898

[4] D. Knoll et al., 16th Topical Meeting on Silicon Monolithic Integrated Circuits in RF Systems, $\mathrm{p}$ 46 (2016), doi: 10.1109/SIRF.2016.7445464.

[5] X. Fan, et al., Opt. Expr. 26, 22944 (2018), doi: 10.1364/OE.26.022944

[6] N. Klimov, et al., Sensors \& Transducers, 191, 63 (2015)

[7] H.Xu et al., Opt. Express 22, 3098 (2014), doi: 10.1364/OE.22.003098

[8] W. Bogaerts et al., Laser Photonics Rev. 6, 47 (2012), doi: 10.1002//por.201100017

[9] D. Grobnic, et al., Proc. SPIE 5855, 17th International Conference on Optical Fibre Sensors, (2005), doi:10.1117/12.623592 